Wassim El-Khatib ${ }^{1}$

Université Libanaise (Liban)

Conseil de l'éducation créative

(Finlande)

\section{Les attitudes des enseignants à l'égard des devoirs scolaires au Liban}

Teachers' attitudes towards homework in Lebanon

doi: $10.18162 / f p .2021 .610$

\section{$\mathrm{R}$ ésumé}

Cet article vise à examiner les attitudes des enseignants de français et en français à l'égard des devoirs scolaires dans les écoles primaires au Liban, dans les deux secteurs public et privé. La méthodologie adoptée s'est appuyée sur le recueil et l'analyse des données issues d'un questionnaire remis aux enseignants. Les résultats montrent l'attachement des enseignants aux pratiques des devoirs scolaires, mais révèlent en même temps un manque de consensus autour de cette activité qui pourrait différer d'un établissement à un autre, ou encore, d'un cycle à un autre.

Mots-clés

Devoirs scolaires, attitudes des enseignants, système scolaire libanais

Abstract

This article aimed at investigating teachers' attitudes of French language and other subjects taught in French towards homework in both public and private primary schools of Lebanon. Data were collected through analysis of a questionnaire sent to teachers. The findings indicate teacher's attachment to school homework and reveal at the same time a lack of consensus on the practice of this activity that may differ between schools and

levels.

\section{Keywords}

Homework, teachers' attitudes, Lebanese educational system.
La pratique des devoirs scolaires suscite des avis contradictoires. Cette pratique est à la fois désirée et rejetée, nécessaire et inutile, efficace et inefficace, sécurisante et source de tension (Bonascio, 2015; Glasman, 2004). Les politiques vis-à-vis des devoirs scolaires varient d'un pays à l'autre, mais la tendance générale est à l'allégement (Cooper, 2015), voire à la suppression des devoirs à faire à domicile (Kralovec, 2014; Kralovec et Buell, 2001).

Au Liban, la restructuration du système éducatif en 1997 a abouti à des modifications qui ont eu des répercussions sur les conditions de travail des enseignants (Hoyek, 2004). La réforme du système éducatif, qui a débouché sur des programmes toujours en vigueur, ne fait aucune allusion à l'organisation des devoirs. À noter également que les programmes de formation initiale des enseignants dispensés à la Faculté de Pédagogie ${ }^{2}$ au Liban n'abordent pas cette question d'une façon fort explicite. Cette dernière est laissée aux soins des dirigeants des établissements scolaires qui accordent une importance capitale à cette pratique. Les devoirs scolaires sont considérés, en effet, comme un moyen de développer les compétences des élèves et d'en évaluer l'acquisition (Ghssaibi, 2017). C'est pourquoi on donne trop de devoirs, on en donne trop tôt et on en donne surtout de trop difficiles. Dans une étude à large échelle initiée par le CRDP, 67,1 $\%$ des élèves libanais trouvent les devoirs scolaires difficiles et même très difficiles (Hatab, 2013, p. 139). Dans cette même étude, 59,3 \% des élèves affirment passer 1 à 3 heures par jour à faire les devoirs et $12,6 \%$ d'entre eux passent même plus de 3 heures quotidiennement à accomplir cette tâche (Ibidem, p. 127). Cette étude conclut que les difficultés rencontrées sur le plan de la charge de travail à domicile constituent un des facteurs d'échec et de décrochage scolaires. Cet 
attachement aux devoirs scolaires au Liban ne laisse pas l'opinion publique indifférente et la presse commence à s'emparer de cette question. Le quotidien local Al-Akhbar y consacre un dossier dont le titre est très évocateur et traduit le malaise à l'égard de ce sujet, soit «Quand les devoirs scolaires deviennent un champ de bataille quotidien » (El-Hage, 2017).

Les études privilégiées sur les devoirs scolaires abordent différentes problématiques dont les principales sont : l'impact de l'engagement des parents dans les devoirs scolaires sur l'amélioration des performances académiques des élèves (Wilder, 2014; Jeyens, 2007; Hill et craft, 2003; Fan et Chen, 2001; Roderique, T. W et al., 1994), le lien entre les devoirs et les résultats scolaires (Murillo et Martinez- Garrido, 2014), l'importance d'expliciter le but du devoir scolaire (Rosario, J. et al., 2018) et l'(in)utilité des devoirs scolaires (Kralovec et Buell, 2001; Cooper 2015).

Le présent article rend compte d'un travail de recherche s'intéressant à la dernière problématique, à savoir l'importance accordée aux devoirs scolaires et les attitudes des enseignants à l'égard de cette pratique.

Dans la littérature scientifique consacrée à ce sujet, on distingue deux positionnements différents et même opposés. Le premier reconnaît aux devoirs scolaires leur contribution, dans certaines conditions, à la réussite des élèves. La synthèse de H. Cooper, J.C Robinson, et E. A Patall (2006) sur les recherches effectuées entre 1987 et 2003 montre qu'il existe une corrélation positive entre les devoirs scolaires et la réussite des élèves dans les grandes classes (G.7- G.12). Cette corrélation positive, selon la même étude, est moins évidente dans les classes élémentaires. La synthèse de Cooper et ses collègues révèle également qu'il n'y a pas de corrélation positive entre le volume horaire réservé aux devoirs et la réussite des élèves et recommande la « règle de 10 minutes » (10-minutes rule). Cette recommandation a été défendue par l'association de l'éducation nationale aux États-Unis (Ciza, 2019). Le second positionnement met en évidence l'inutilité des devoirs scolaires, voire leurs effets négatifs sur les élèves. Kralovec et Buell (2001, p. 39) considèrent que les devoirs scolaires comme facteur ayant " contribué d'une façon si spectaculaire au décrochage scolaire » (Kralovec et Buell, 2001, p. 39). Kalish, N., et Bennett, S. (2007) intentent un vrai procès contre les devoirs scolaires dans "The case against homework: How homework is hurting our children and what we can do about it ». Dans un livre problématique, sans doute, Kalish et Bennett pointent du doigt l'inutilité de cette pratique et ses effets néfastes sur les élèves. Kalish et Bennett révèlent, par ailleurs, que les enseignants ne sont pas formés à donner des devoirs. Cette lacune est soulevée également par Robin et al. (2015) qui précisent que la formation aux devoirs n'est pas souvent abordée dans les curriculums de formation initiale des enseignants et dans la réforme des programmes.

$\mathrm{Vu}$ les problèmes que soulève la question des devoirs à domicile, la recherche scientifique s'intéresse aux attitudes des principaux acteurs concernés, notamment les enseignants, à l'égard de cette pratique (Magalhaes, 2014; Meister 2014; Hong, Wan, et Peng, 2011; Brock et al., 2007).

À la lumière de ce qui précède, la présente étude vise donc à examiner les attitudes des enseignants à l'égard des devoirs scolaires dans les écoles primaires au Liban, d'autant plus que les études sur les devoirs scolaires en général et celles relatives aux conceptions des enseignants à l'égard de cette pratique, en particulier, sont quasiment inexistantes.

2 - Formation et profession 29(1), 2021 


\section{Méthodologie de la recherche}

La méthodologie de la présente recherche consiste à s'enquérir des attitudes des enseignants de français et en français dans les écoles publiques et privées. Il s'agit donc d'une méthode descriptive exploratoire combinant à la fois une démarche quantitative et qualitative.

\section{Outil de la recherche}

Compte tenu des objectifs et des contraintes de cette recherche, un questionnaire élaboré par Fawzy (2012) a été adopté. Ce questionnaire, conçu au départ par Brock et al. (2007) et Cameron et Bartel (2009), a été adapté pour tenir compte de la particularité du contexte: traduction en français, modification des items et ajout de deux questions ouvertes visant à recueillir des données qualitatives. La première concerne les finalités des devoirs et la seconde est une question qui porte sur les remarques et les suggestions que les participants désirent formuler. La cohérence interne du questionnaire a été mesurée à l'aide du test de validité : alpha de Cronbach. Le résultat de l'ensemble des domaines (0,61 à 0,87 ) est jugé satisfaisant étant donné que la recherche est descriptive exploratoire.

Le questionnaire est formé de 30 items répartis en six domaines intitulés : Finalités des devoirs 1 (4 items), Finalités des devoirs 2 (3 items), Mes propres croyances sur les devoirs scolaires 1 (5 items), Mes propres croyances sur les devoirs scolaires 2 (7 items), Engagements des parents dans la pratique des devoirs (6 items), Pratiques des devoirs en classe (5 items). La durée approximative pour répondre au questionnaire est de 7 à 10 minutes.

En vue de faciliter l'administration du questionnaire et atteindre l'ensemble de l'échantillon, il a été mis en ligne sur le site : http://www.questenligne.com/

\section{Échantillonnage}

L'échantillon de la recherche est composé des enseignants de français et en français des deux secteurs public et privé, tous cycles confondus, ayant bien voulu participer à la recherche. En raison des mesures de confinement dues à la pandémie COVID-19, et compte tenu des limites de la recherche, le questionnaire n'a pas pu être remis à tous les enseignants concernés dont le nombre est estimé à plus de 10000 (alef-lbn.fipf.org). L'échantillon s'est limité aux enseignants adhérant aux deux associations des enseignants de français : ALEF et ANEFL et aux enseignants du réseau de L'AEFE dont le nombre total s'élève à 1290 . Il convient de préciser que le questionnaire, mis en ligne, a été remis aux enseignants à partir d'un lien diffusé sur les réseaux sociaux des enseignants. Ainsi, 200 réponses ont été obtenues. L'échantillon est donc loin d'être représentatif, mais le nombre de réponses obtenu et la distribution de l'échantillon ont permis de réaliser les analyses quantitatives et qualitatives nécessaires. En effet, la répartition de l'échantillon en ce qui concerne le type d'école, le cycle et l'âge, est homogène. Pour ce qui est du type d'école, les réponses sont distribuées ainsi : écoles publiques : $39,5 \%$; écoles privées à programme libanais : 32,0 \%, écoles privées à programmes français : 28,5\%. Concernant le cycle, les résultats sont les suivants : cycle primaire : $31,5 \%$, cycle complémentaire (collège) : 33,5 \%, Lycée : 35,0 \%. En matière d'âge, les participants sont répartis ainsi : 23,5 \% (22 ans à 32 ans), 29,5\% (33 ans à 43 ans), 31,0 \% (44 ans à 54 ans), 16,0 \% (55 ans à 64 ans). Certains résultats, paraissant 
d'emblée incohérents, reflètent les particularités de la population de la recherche. C'est le cas, par exemple du genre des participants : 95,5\% des femmes et 4,5\% des hommes. Le métier d'enseignant au Liban a tendance à la féminisation, et ce, dans les différents secteurs (Rapport PASEC Liban, 2012, p. 20). C'est le cas également des matières enseignées. La majorité des participants enseigne le français : $66 \%$, ce qui paraît tout à fait logique étant donné que le questionnaire s'adresse principalement aux enseignants de français et en français.

\section{Présentation et analyse des résultats}

\section{Analyse quantitative}

Avant de procéder à la présentation et à l'analyse quantitative des résultats, il convient de préciser que les données issues des questions fermées de type Likert à 5 items ont été consignées dans le logiciel SPSS et ont fait l'objet d'analyses statistiques de deux types : statistiques descriptives usuelles (pourcentage, moyenne, écart-type) et statistiques inférentielles, notamment le Coefficient de contingence (c) pour vérifier les liens entre les variables indépendantes (âge, type d'école, cycle, etc.) et les différentes dimensions étudiées. Le choix du test de contingence a été déterminé par la nature de variables retenues. Il est à noter que, dans la présente recherche, les modalités de réponse de type échelle de Likert et de type ordinale sont rangées dans la catégorie de "variables nominales " (ChanvrilLigneel et Le Hay, 2014, p. 47). Pour ne pas alourdir le texte de l'analyse, les résultats du coefficient de contingence ne vont être évoqués que dans le cas où ces derniers se révèlent significatifs. À noter également que pour des raisons d'ordre pratique, les abréviations suivantes vont être employées dans les tableaux de présentation des résultats de l'analyse quantitative : FED (Fortement En Désaccord), ED (En Désaccord), N (Neutre), EA (En Accord), FEA (Fortement En Accord).

\section{Résultats du premier domaine « Finalités des devoirs 1 »}

Les résultats du premier domaine (tableau 1) révèlent que les enseignants nont pas tous la même attitude vis-à-vis des différentes finalités composant ce domaine. Ce qui semble constituer l'unanimité parmi les enseignants, c'est l'item $2:$ «Je donne des devoirs afin que les élèves révisent la leçon donnée en classe ». 


\section{Tableau 1}

Résultats du premier domaine

\begin{tabular}{lcccccc} 
Items & $\begin{array}{c}\text { Moyenne } \\
\text { (Écart-type) }\end{array}$ & $\begin{array}{c}\text { FED } \\
\%\end{array}$ & $\begin{array}{c}\text { ED } \\
\%\end{array}$ & $\begin{array}{c}\text { N } \\
\%\end{array}$ & $\begin{array}{c}\text { EA } \\
\%\end{array}$ & $\begin{array}{c}\text { FEA } \\
\%\end{array}$ \\
\hline $\begin{array}{l}\text { Q.1 Je donne des devoirs afin que les élèves } \\
\text { préparent une nouvelle leçon. }\end{array}$ & $\begin{array}{c}2,7 \\
(1,23)\end{array}$ & 20 & 24,5 & 19 & 31 & 5,5 \\
\hline $\begin{array}{l}\text { Q.2 Je donne des devoirs afin que les élèves } \\
\text { révisent la leçon donnée en classe. }\end{array}$ & $\begin{array}{c}4,05 \\
(0,82)\end{array}$ & 1,5 & 5,5 & 5,5 & 61 & 28,5 \\
\hline $\begin{array}{l}\text { Q.3 Je donne des devoirs afin que les élèves } \\
\text { mémorisent la leçon expliquée en classe. }\end{array}$ & $\begin{array}{c}3,37 \\
(1,21)\end{array}$ & 9,5 & 18,5 & 12,5 & 44,5 & 15 \\
\hline $\begin{array}{l}\text { Q.4 Je donne des devoirs pour encourager la } \\
\text { collaboration entre élèves. }\end{array}$ & $\begin{array}{c}3,29 \\
(1,21)\end{array}$ & 6 & 18 & 29 & 35 & 12 \\
\hline
\end{tabular}

Les enseignants semblent développer la même attitude à l'égard de la pratique des devoirs pour « mémoriser la leçon expliquée » (item 3), mais avec un degré d'unanimité moindre. Les pourcentages relatifs à cet item demeurent toutefois positifs : 44,5\% pour « Entièrement en accord » et $15 \%$ pour "Fortement en accord ». L'hétérogénéité des résultats pourrait être due aux pratiques des devoirs scolaires dans les différentes écoles et dans les différents cycles. En effet, les résultats du Coefficient de contingence (c) sont statistiquement significatifs pour ce qui est du lien entre le type d'école et le cycle et le domaine :

Type d'école*Finalités des devoirs scolaires $1:$ Sig. $=0,007$

Cycle* Finalités des devoirs scolaires $1:$ Sig. $=0,014$.

\section{Résultats du $2^{e}$ domaine « Finalités des devoirs 2 »}

Les résultats du deuxième domaine (tableau 2) montrent que les enseignants ne sont pas favorables aux pratiques des devoirs à des fins non liées à l'apprentissage. Les statistiques sont très significatives à cet égard, notamment pour les deux premiers items. Les pourcentages s'élèvent à 88,5\% pour la somme de "Fortement en désaccord » et " en désaccord ", dans le cas du premier item : "Je donne des devoirs pour punir les élèves ", et à $73 \%$ pour la somme de « Fortement en désaccord » et « en désaccord », dans le cas du deuxième item : "Je donne des devoirs pour plaire aux parents qui en sont demandeurs ». 


\section{Tableau 2}

Résultats du $2^{e}$ domaine

\begin{tabular}{|c|c|c|c|c|c|c|}
\hline Items & $\begin{array}{l}\text { Moyenne } \\
\text { (Écart-type) }\end{array}$ & $\begin{array}{c}\text { FED } \\
\%\end{array}$ & $\begin{array}{l}\mathrm{ED} \\
\%\end{array}$ & $\begin{array}{l}\mathrm{N} \\
\%\end{array}$ & $\begin{array}{l}\mathrm{EA} \\
\%\end{array}$ & $\begin{array}{c}\text { FEA } \\
\%\end{array}$ \\
\hline Q.1 Je donne des devoirs pour punir les élèves. & $\begin{array}{c}1,47 \\
(0,83) \\
\end{array}$ & 69 & 19,5 & 8,5 & 1,5 & 1,5 \\
\hline $\begin{array}{l}\text { Q.2 Je donne des devoirs pour plaire aux parents qui } \\
\text { en sont demandeurs. }\end{array}$ & $\begin{array}{c}1,87 \\
(1,08)\end{array}$ & 44 & 29 & 23 & 4 & 28,5 \\
\hline $\begin{array}{l}\text { Q.3 Je donne des devoirs pour être en conformité avec } \\
\text { le règlement de l'école. }\end{array}$ & $\begin{array}{c}2,69 \\
(1,21)\end{array}$ & 17 & 26 & 29 & 26,5 & 1,5 \\
\hline
\end{tabular}

Toutefois, les résultats du troisième item : "Je donne des devoirs pour être en conformité avec le règlement de l'école » sont plus dispersés. L'écart-type est de 1,21 et la moyenne est de 2,69. La dispersion des réponses pourrait être due, à notre avis, à l'appréhension des enseignants à répondre à une question ayant trait au règlement des écoles, et ce, malgré l'anonymat du questionnaire. Il est à noter que les résultats du coefficient de contingence ne sont pas statistiquement significatifs, ce qui confirme notre constat et montre, par ailleurs, qu'il n'y pas de lien entre les variables indépendantes (âge, type d'école, cycle, etc.) et la dimension étudiée dans ce domaine.

\section{Résultats du $3^{e}$ domaine «Mes propres croyances sur les devoirs scolaires 1 »}

Les résultats du troisième domaine (tableau 3) montrent que les enseignants sont favorables à l'idée que les devoirs scolaires permettent de développer des compétences et des valeurs de base chez les apprenants et constituent une aide à l'apprentissage en général.

\section{Tableau 3}

Résultats du $3^{e}$ domaine

\begin{tabular}{lcccccc} 
Items & $\begin{array}{c}\text { Moyenne } \\
\text { (Écart-type) }\end{array}$ & $\begin{array}{c}\text { FED } \\
\%\end{array}$ & $\begin{array}{c}\text { ED } \\
\%\end{array}$ & $\begin{array}{c}\text { N } \\
\%\end{array}$ & $\begin{array}{c}\text { EA } \\
\%\end{array}$ & $\begin{array}{c}\text { FEA } \\
\%\end{array}$ \\
\hline $\begin{array}{l}\text { Q.1 Je crois que les devoirs permettent aux élèves de } \\
\text { développer de bonnes habitudes telles que la gestion } \\
\text { du temps et l'organisation }\end{array}$ & $\begin{array}{c}3,61 \\
(1,04)\end{array}$ & 6 & 10,5 & 13 & 57 & 13,5 \\
\hline $\begin{array}{l}\text { Q.2 Je crois que les devoirs inculquent des valeurs } \\
\text { importantes telles que l'autonomie, la responsabilité et } \\
\text { la persévérance. }\end{array}$ & $\begin{array}{c}3,79 \\
(0,93)\end{array}$ & 4 & 8 & 8 & 65 & 15 \\
\hline $\begin{array}{l}\text { Q.3 Je crois que les devoirs aident les élèves à atteindre } \\
\text { de meilleurs résultats scolaires. }\end{array}$ & $\begin{array}{c}3,39 \\
(1,09)\end{array}$ & 6 & 19,5 & 14 & 50 & 10,5 \\
\hline $\begin{array}{l}\text { Q.4 Je crois que les devoirs aident les élèves à } \\
\text { comprendre que l'apprentissage se fait aussi en dehors } \\
\text { de l'école. }\end{array}$ & $\begin{array}{c}3,51 \\
(1,02)\end{array}$ & 5 & 13 & 18,5 & 53 & 10,5 \\
\hline $\begin{array}{l}\text { Q.5 Je crois que les devoirs développentles compétences } \\
\text { acquises à l'école }\end{array}$ & $\begin{array}{c}3,76 \\
(0,94)\end{array}$ & 3,5 & 10,5 & 6,5 & 65,5 & 14 \\
& & & & & & \\
\hline
\end{tabular}


Par ailleurs, les résultats du coefficient de contingence ne sont pas statistiquement significatifs, ce qui signifie qu'il n'y pas de lien entre les variables indépendantes (âge, type d'école, cycle, etc.) et la dimension étudiée dans ce domaine.

\section{Résultats du $4^{e}$ domaine «Mes propres croyances sur les devoirs scolaires 2 »}

Les résultats du quatrième domaine (tableau 4) montrent que les avis sont départagés quant aux effets négatifs des devoirs scolaires. Seuls, les items 5 et 6 reçoivent des valeurs assez importantes. D'autres valeurs assez importantes méritent d'être soulignées. C'est le cas du $3^{\mathrm{e}}$ et $\mathrm{du} 4^{\mathrm{e}}$ items qui reçoivent respectivement $42 \%$ et $43 \%$ pour la somme de «En accord » et «Fortement en accord ». Il nous paraît significatif qu'un bon nombre d'enseignants pense que les devoirs scolaires " privent les enfants du temps à passer avec leurs familles et de leurs loisirs » et qu'ils «causent du stress et des conflits dans la famille ».

\section{Tableau 4}

Résultats du $4^{e}$ domaine

\begin{tabular}{lcccccc} 
Items & $\begin{array}{c}\text { Moyenne } \\
\text { (Écart-type) }\end{array}$ & $\begin{array}{c}\text { FED } \\
\%\end{array}$ & $\begin{array}{c}\text { ED } \\
\%\end{array}$ & $\begin{array}{c}\text { N } \\
\%\end{array}$ & $\begin{array}{c}\text { EA } \\
\%\end{array}$ & $\begin{array}{c}\text { FEA } \\
\%\end{array}$ \\
\hline $\begin{array}{l}\text { Q.1 Je crois que les devoirs démotivent les élèves à } \\
\text { apprendre. }\end{array}$ & $\begin{array}{c}2,74 \\
(1,01)\end{array}$ & 7 & 42 & 25,5 & 21 & 4,5 \\
\hline $\begin{array}{l}\text { Q.2 Je crois que les devoirs causent de la fatigue physique } \\
\text { et de l'insomnie. }\end{array}$ & $\begin{array}{c}2,88 \\
(1,10)\end{array}$ & 5,5 & 42 & 20 & 24 & 8,5 \\
\hline $\begin{array}{l}\text { Q.3 Je crois que les devoirs privent les enfants du temps à } \\
\text { passer avec leurs familles et de leurs loisirs. }\end{array}$ & $\begin{array}{c}3,03 \\
(1,09)\end{array}$ & 6 & 38,5 & 12,5 & 32 & 11 \\
\hline $\begin{array}{l}\text { Q.4 Je crois que les devoirs causent du stress et des conflits } \\
\text { dans la famille. }\end{array}$ & $\begin{array}{c}3,10 \\
(1,17)\end{array}$ & 4,5 & 33 & 20,5 & 32 & 10 \\
\hline $\begin{array}{l}\text { Q.5 Je crois que les devoirs empêchent les enfants de nouer } \\
\text { des amitiés. }\end{array}$ & $\begin{array}{c}2,55 \\
(1,001)\end{array}$ & 7,5 & 53,5 & 22 & 10,5 & 6,5 \\
\hline $\begin{array}{l}\text { Q.6 Je crois que les devoirs augmentent l'écart entre les } \\
\text { bons élèves et ceux qui sont moins performants. }\end{array}$ & $\begin{array}{c}2,56 \\
(0,99)\end{array}$ & 8 & 51,5 & 21,5 & 14 & 5 \\
\hline Q.7 Je crois que les devoirs peuvent être annulés. & $\begin{array}{c}2,99 \\
(1,17)\end{array}$ & 8 & 35,5 & 16 & 30,5 & 10 \\
& & & & & \\
\hline
\end{tabular}

Un autre résultat significatif mériterait d'être souligné, à notre sens. Il s'agit du dernier item : « Je crois que les devoirs peuvent être annulés ». Malgré la dispersion des réponses, il nous paraît évident que les devoirs scolaires commencent à être remis en question. Pour ce qui est des résultats du coefficient de contingence, ils ne sont pas statistiquement significatifs. 


\section{Résultats du $5^{e}$ domaine «Engagements des parents dans la pratique des devoirs »}

Les résultats du cinquième domaine (tableau 5) révèlent que les enseignants ne développent pas les mêmes attitudes à l'égard de l'engagement des parents dans la pratique des devoirs. Ils semblent, d'emblée, plutôt défavorables à l'implication des parents dans l'éducation de leurs enfants. Ils ne s'attendent pas non plus à ce que « les parents aident leurs enfants à faire leurs devoirs ».

\section{Tableau 5}

Résultats du $5^{e}$ domaine

\begin{tabular}{lcccccc} 
Items & $\begin{array}{c}\text { Moyenne } \\
\text { (Écart-type) }\end{array}$ & $\begin{array}{c}\text { FED } \\
\%\end{array}$ & $\begin{array}{c}\text { ED } \\
\%\end{array}$ & $\begin{array}{c}\text { N } \\
\%\end{array}$ & $\begin{array}{c}\text { EA } \\
\%\end{array}$ & $\begin{array}{c}\text { FEA } \\
\%\end{array}$ \\
\hline $\begin{array}{l}\text { Q.1 Je donne des devoirs parce que je pense qu'il } \\
\text { est important d'impliquer les parents dans l'éducation } \\
\text { de leurs enfants. }\end{array}$ & $\begin{array}{c}2,55 \\
(1,09)\end{array}$ & 17 & 39 & 18 & 24 & 2 \\
\hline $\begin{array}{l}\text { Q.2 Je crois que les différentes techniques d'instruction } \\
\text { utilisées par les parents perturbent les enfants. }\end{array}$ & $\begin{array}{c}3,64 \\
(0,93)\end{array}$ & 2,5 & 10 & 23 & 49,5 & 15 \\
\hline $\begin{array}{l}\text { Q.3 Je m'attends à ce que les parents aident leurs enfants } \\
\text { à faire leurs devoirs. }\end{array}$ & $\begin{array}{c}2,09 \\
(0,96)\end{array}$ & 29 & 45,5 & 14 & 10,5 & 1 \\
\hline $\begin{array}{l}\text { Q.4 Je tiens à savoir si les enfants reçoivent une aide } \\
\text { adéquate à la maison. }\end{array}$ & $\begin{array}{c}3 \\
(1,09)\end{array}$ & 10,5 & 22 & 23 & 40,5 & 4 \\
\hline $\begin{array}{l}\text { Q.5 Je fournis des explications aux parents pour la supervision } \\
\text { des devoirs. }\end{array}$ & $\begin{array}{c}3 \\
(1,08)\end{array}$ & 11,5 & 24,5 & 28 & 32,5 & 3,5 \\
\hline $\begin{array}{l}\text { Q.6 Je fournis aux parents un moyen de communication pour } \\
\text { discuter des devoirs. }\end{array}$ & $\begin{array}{c}3 \\
(1,08)\end{array}$ & 12 & 25,5 & 24,5 & 35,5 & 2,5
\end{tabular}

Ils croient, par ailleurs, que « les différentes techniques d'instruction utilisées par les parents perturbent les enfants ». Pour ce qui est des items 4, 5 et 6, les résultats ne permettent pas de déceler une tendance bien déterminée chez les enseignants. Il est à noter, en dernier lieu, que les résultats du coefficient de contingence ne sont pas statistiquement significatifs. 


\section{Résultats du $6^{e}$ domaine «Pratiques des devoirs en classe »}

Les résultats du dernier domaine relatif aux pratiques des devoirs en classe (tableau 6), permettent de déceler une certaine homogénéité au niveau des 3 premiers items.

\section{Tableau 6}

Résultats du $6^{e}$ domaine

\begin{tabular}{lcccccc} 
Items & $\begin{array}{c}\text { Moyenne } \\
(\text { Écart-type) }\end{array}$ & $\begin{array}{c}\text { FED } \\
\%\end{array}$ & $\begin{array}{c}\text { ED } \\
\%\end{array}$ & $\begin{array}{c}\text { N } \\
\%\end{array}$ & $\begin{array}{c}\text { EA } \\
\%\end{array}$ & $\begin{array}{c}\text { FEA } \\
\%\end{array}$ \\
\hline Q.1 Les enfants notent leurs devoirs sur l'agenda. & $\begin{array}{c}1,74 \\
(1,02)\end{array}$ & 56 & 23,5 & 12,5 & 6 & 2 \\
\hline Q.2 Je vérifie que les devoirs sont remis. & $\begin{array}{c}1,6 \\
(0,89)\end{array}$ & 61 & 23 & 12,5 & 2 & 1,5 \\
\hline $\begin{array}{l}\text { Q.3 Je donne une rétroaction (corrections, } \\
\text { remarques, etc.) sur les devoirs. }\end{array}$ & $\begin{array}{c}1,58 \\
(0,84)\end{array}$ & 60 & 26 & 10 & 3,5 & 0,5 \\
\hline $\begin{array}{l}\text { Q.4 Les devoirs sont inclus dans l'évaluation. } \\
\text { (1,37 }\end{array}$ & 7 & 20,5 & 27,5 & 18,5 & 26,5 \\
\hline $\begin{array}{l}\text { Q.5 Les enfants sont punis pour ne pas avoir } \\
\text { remis leurs devoirs. }\end{array}$ & $\begin{array}{c}3,4 \\
(1,09)\end{array}$ & 3,5 & 17 & 35,5 & 23,5 & 20,5
\end{tabular}

Les résultats du dernier domaine relatif aux pratiques des devoirs en classe (tableau 6), permettent de déceler une certaine homogénéité en ce qui concerne les 3 premiers items. Les statistiques sont significatives à ce niveau. Ces résultats paraissent étonnants et sont, à notre avis, le signe d'une certaine ambivalence chez les enseignants. Donner des devoirs sans s'assurer que ces derniers soient notés sur l'agenda, ou remis et sans fournir une rétroaction aux apprenants remet en question, à notre sens, les finalités de cette pratique. Les résultats des items 4, 5 sont dispersés, mais avec une concentration des réponses «neutres »: $35 \%$, dans le cas de l'item 5 et $27 \%$, dans le cas de l'item 4. Cette neutralité est difficile à interpréter, mais pourrait révéler une certaine réticence à évoquer un sujet sensible tel que l'évaluation. Pour ce qui est des résultats du coefficient de contingence, ils ne sont pas statistiquement significatifs.

\section{Analyse qualitative}

Cette partie fait état de l'analyse qualitative des remarques recensées qui sont au nombre de 67 pour la première question portant sur les raisons de donner des devoirs et 54 pour la deuxième concernant les remarques et suggestions à faire au sujet des devoirs scolaires. L'analyse appliquée est une analyse de contenu catégorielle (Bardin, 1997). La définition des catégories s'est basée sur deux sources : «le document lui-même » et une « certaine connaissance du domaine » (Grawitz, 2001, p.617)

L'analyse de la première question a permis de distinguer onze catégories (tableau 7). 


\section{Tableau 7}

Analyse de la question portant sur les finalités des devoirs scolaires

Catégorie Nombre de réponses

1. Développer des habiletés cognitives : mémorisation, application, entrânement, 45 consolidation, réinvestissement et évaluation des acquis.

\begin{tabular}{lll}
\hline 2. & Refuser de donner des devoirs & 6 \\
\hline 3. & Développer l'autonomie & 3 \\
\hline 4. & Simplifier le devoir & 3 \\
\hline 5. & Faire de la recherche & 3 \\
\hline 6. & Enrichissement, se cultiver & 2 \\
\hline 7. & Communiquer les apprentissages aux parents & 1 \\
\hline 8. & Différencier les devoirs & 1 \\
\hline 9. & Responsabiliser l'élève & 1 \\
\hline 10. & Préparer une nouvelle leçon & 1 \\
\hline 11. & Faciliter l'explication & 1
\end{tabular}

Mais la catégorie qui a reçu le plus de récurrences est celle de « développement des habiletés cognitives » (45 récurrences). Les autres catégories ont reçu de faibles récurrences. Elles concernent principalement le refus de donner des devoirs, le développement de l'autonomie de l'élève, l'allégement des devoirs, etc.

En ce qui concerne l'analyse de la deuxième question portant sur les remarques et suggestions à faire au sujet des devoirs scolaires, huit catégories ont été retenues (tableau 8).

\section{Tableau 8}

Analyse de la question portant sur les remarques et les suggestions au sujet des devoirs scolaires

Catégorie Nombre de réponses

\begin{tabular}{lll}
\hline 1. & Opposition aux devoirs surchargés et traditionnels au cycle primaire & 28 \\
\hline 2. & Organisation des devoirs (quantité, durée, contenu et technique) & 10 \\
\hline 3. & Nécessité des devoirs & 9 \\
\hline 4. & Inutilité des devoirs & 3 \\
\hline 5. & L'irrespect du devoir par les élèves & 1 \\
\hline 6. & Devoir fait en classe & 1 \\
\hline 7. & Des parents s'opposant aux devoirs & 1 \\
\hline 8. & Réforme des programmes & 1
\end{tabular}


La catégorie qui a reçu le plus de récurrences est la première (28 récurrences). Elle porte sur le refus des devoirs surchargés au cycle primaire. Viennent ensuite la deuxième catégorie ayant trait à l'organisation des devoirs (10 récurrences) et la troisième catégorie relative à la nécessité des devoirs (9 occurrences). Les catégories ayant de faibles récurrences concernent principalement les problèmes liés à la pratique des devoirs tels que l'irrespect du devoir par l'élève, l'opposition des parents aux devoirs, le souhait de faire le devoir en classe et de réorganiser cette pratique dans la réforme des programmes.

Les résultats de l'analyse de contenu rejoignent en gros ceux de l'analyse quantitative. Les enseignants pensent que les devoirs scolaires permettent de développer des compétences de base et venir en aide à l'apprentissage, mais soulignent, en même temps, la nécessité de les réorganiser et de les alléger. L'analyse de contenu révèle que certains enseignants semblent mettre en question, comme c'est le cas de l'analyse quantitative, toute la pratique des devoirs scolaires.

\section{Discussion des résultats}

Les résultats de l'enquête révèlent, en général, que les enseignants développent une attitude favorable à l'égard des devoirs scolaires. De leur point de vue, les devoirs scolaires permettent de développer des compétences et des valeurs de base chez les apprenants et constituent une aide à l'apprentissage en général. Leur positionnement semble s'accorder davantage avec celui attribuant aux devoirs scolaires des effets bénéfiques sur l'apprentissage et la performance des élèves (Cooper, 2005 et 2014). Toutefois, force est de constater qu'une tendance à remettre en question cette pratique commence à se faire sentir chez une partie des enseignants qui pense que les devoirs devraient être allégés et même abolis. Cette hétérogénéité des opinions, constatée à plus d'endroits, est le signe que la pratique des devoirs scolaires est à la fois « désirée et rejetée, nécessaire et inutile, efficace et inefficace, sécurisant et source de tension » (Favre et Steffen, 1988, cités par Glasman et Besson, 2005, p. 16). Or, cette hétérogénéité devrait, à notre avis, soulever la question de la formation des enseignants qui n'est pas souvent abordée dans les curriculums de formation initiale des enseignants et dans la réforme des programmes (Robin et al., 2015).

Les résultats de l'enquête montrent, par ailleurs, que les enseignants paraissent défavorables à l'implication des parents dans les devoirs à domicile. Ces résultats vont à l'encontre des études menées par Fan et Chen (2001), Jeyens (2007), Wilder (2014) et Alexander et Entwistle (1996) confirmant que l'implication des parents est un des principaux prédicateurs de la réussite scolaire. La réticence des enseignants pourrait être due à une idée répandue que les parents ne sont pas « des professionnels de la transmission des savoirs » et que toute tâche d'enseignement doit être réservée aux enseignants (Gruss, 2019, p. 12).

Toujours est-il que certains résultats paraissent étonnants et difficiles à interpréter. Les enseignants sont, en effet, défavorables à l'idée que les devoirs scolaires doivent être notés sur l'agenda, vérifiés après être mis et faire l'objet d'une rétroaction. Ces résultats vont, bien entendu, à l'encontre des études évoquées dans la littérature scientifique. Murillo et Martinez-Garrido (2014) affirment que l'amélioration des performances académiques des élèves se trouve en étroite relation avec la façon dont l'enseignant évalue les devoirs en classe. De même, l'étude menée par Rosario et ses collaborateurs (2018) montre que le fait de communiquer aux élèves l'utilité du devoir leur permet de promouvoir leur engagement dans 
la tâche et de renforcer leur sentiment d'auto- efficacité et de motivation. Mais comment interpréter dès lors le manque des enseignants à des activités d'une extrême importance sur le plan pédagogique ? Si le fait de ne pas vérifier que les devoirs soient notés sur l'agenda pouvait être attribué au souci de développer l'autonomie des élèves (Lacroix, 2012), il serait très difficile de justifier le manque de suivi de la part de l'enseignant. On est en droit de se demander si les enseignants perçoivent cette activité tout simplement comme une corvée. "Je ne crois pas aux bienfaits des devoirs mais je me sens obligée d'en donner ", affirme une enseignante, dans une enquête sur les devoirs scolaires menée par Thlaoui et Biesemans (2020). En tout état de cause, ces résultats confirment, à nos yeux, la nécessité de la formation des enseignants aux devoirs scolaires et la définition d'une politique éducative prenant en considération des aspects souvent négligés des pratiques enseignantes tels que les devoirs à domicile.

\section{Conclusion}

Cette recherche portant sur les devoirs scolaires du point de vue des enseignants libanais a permis, à notre sens, d'éclairer d'un jour nouveau une question très peu traitée par la recherche au Liban. Les résultats montrent, d'emblée, que les enseignants libanais sont favorables à l'égard des devoirs scolaires et leur accordent beaucoup d'importance sur le plan pédagogique. Mais les résultats révèlent, également, qu'une tendance à mettre une question cette pratique commence à se faire sentir. Les résultats mettent en évidence, par ailleurs, la pratique des devoirs n'est pas exempte de biais qui soulèvent des questions de taille. Ne pas s'assurer que les devoirs soient notés sur l'agenda de l'élève et s'abstenir d'assurer un suivi aux devoirs amène à s'interroger sur le bien-fondé de cette pratique.

Il incombe d'évoquer, également, dans ce paragraphe conclusif, les limites de cette recherche. En effet, malgré l'importance des résultats obtenus, il demeure difficile de les généraliser à une grande échelle. L'échantillon de cette recherche a concerné uniquement les enseignants de français et en français qui ont bien voulu participer à cette recherche. Par conséquent, l'échantillon est loin d'être représentatif. De même, cette recherche a pris en considération seulement le point de vue des enseignants sans s'intéresser à celui des autres acteurs : les parents, les élèves et les membres du cadre administratif. À noter, en dernier lieu, que pour des contraintes d'ordre technique et matériel, l'outil de recherche utilisé s'est limité à l'administration d'un questionnaire auprès des enseignants.

À la lumière de tout ce qui précède, plusieurs recommandations pourraient être proposées, à notre avis.

Il serait fortement recommandé de repenser la pratique des devoirs scolaires en vue d'une meilleure organisation de son mode de fonctionnement. Les propositions formulées par les enseignants paraissent tout à fait pertinentes : réduire le nombre de devoirs, limiter cette pratique aux cycles supérieurs (collège et lycée), veiller à ce que les consignes soient bien explicitées et que la charge de travail soit à la portée des enfants.

Il paraît plus que nécessaire, également, d'éclaircir le contrat didactique relatif aux devoirs scolaires, voire de l'instaurer dans chaque établissement. Il serait absurde d'attribuer un travail à domicile et de ne pas veiller à ce que ce travail soit remis et évalué.

Il serait bon aussi d'étudier la possibilité d'engager les parents dans cette pratique, non dans le sens d'accentuer les inégalités entre les apprenants, mais dans le but de les réduire et d'assurer un meilleur 
rendement de cette pratique. Il serait nécessaire, à cette fin, que les objectifs des devoirs scolaires et les « règles du jeu » soient clairement explicités aux parents : temps imparti aux devoirs, autonomie de l'apprenant, etc.

Il serait enfin plus que nécessaire de former les enseignants à la pratique des devoirs et définir une politique éducative au sujet des devoirs scolaires à l'instar de certains pays tels que la France et la Belgique. La pratique des devoirs scolaires est étroitement liée à l'approche retenue dans un pays donné et au positionnement éducatif de la «noosphère » pour reprendre le terme de Chevallard (1985). Qu'est-ce qu'un système éducatif cherche à développer chez les apprenants ? Des connaissances ? Des compétences? L'autonomie ? La réflexion critique ? La réponse à ces questions détermine dans une large mesure, à notre sens, la position à prendre face aux devoirs scolaires.

\section{Notes}

1 Wassim El-Khatib, a participé à l'élaboration des programmes de 1997, toujours en vigueur et il est un enseignantchercheur à la Faculté de Pédagogie de l’Université Libanaise depuis 2010.

2 La Faculté de Pédagogie de l’Université Libanaise est le seul organisme chargé de la formation initiale des enseignants.

\section{Liste de références}

Bardin, L. (1977). L'analyse de contenu. Presses Universitaires de France.

Bonasio, R. (2015). La pratique des devoirs en classe et en dehors de la classe [thèse de l'Université Toulouse le Mirail - Toulouse I, France]. archives-ouvertes.fr. https://tel.archives-ouvertes.fr/tel-01538302/document

Brock, C. H., Lapp, D., Flood, J., Fisher, D., \& Han, K. T. (2007). Does homework matter? An investigation of teacher perceptions about homework practices for children from nondominant backgrounds. Urban Education, 42(4), 349-372. https://doi.org/10.1177/0042085907304277

Cameron, L., \& Bartel, L. (2009). The researchers ate the homework: Perspectives of parents and teachers. Education Canada, 49(1), 48-50. https://www.edcan.ca/wp-content/uploads/EdCan-2009-v49-n1-Cameron.pdf

Chanvril-Ligneel, F. et Le Hay, V. (2014). Méthodes statistiques pour les sciences sociales. Ellipses.

Chevallard, Y. (1991). La transposition didactique - Du savoir savant au savoir enseigné( $2^{\mathrm{e}}$ éd.). La Pensée sauvage.

Ciza, E. (2019). Les devoirs scolaires dans les établissements secondaires au Québec : un analyseur-révélateur des conceptions des pratiques enseignantes [Thèse de doctorat de l'Université de Sherbrooke, Québec, Canada]. Savoirs UdeS. https://savoirs.usherbrooke.ca/bitstream/handle/11143/15131/Ciza Ezechiel PhD 2019.pdf? sequence=4\&isAllowed= y

Cooper, H. (2015). The battle over homework: Common ground for administrators, teachers, and parents. Simon and Schuster. DOI: http://dx.doi.org/10.4135/9781483329420

Cooper, H., Robinson, J. C., \& Patall, E. A. (2006). Does homework improve academic achievement? A synthesis of research, 1987-2003. Review of Educational Research, 76(1), 1-62. https:/www.almendron.com/tribuna/wp-content/uploads/2016/02/Does-Homework-Improve-Academic-Achievement.pdf

E1-Hage, F. (2017, 30 octobre). Quand les devoirs scolaires deviennent un champ de bataille quotidien. Al-Achbar. https://al-akhbar.com/Home Page/239847

Fan, X., \& Chen, M. (2001). Parental involvement and students' academic achievement: A meta-analysis. Educational Psychology Review, 13(1), 1-22. DOI: https://doi.org/10.1023/A:1009048817385 
Fawzy, N. (2012). School teacher's perspectives on homework [mémoire de l'Université américaine du Caire, Caire, Egypte]. AUC Knowledge Fountain.

https://fount.aucegypt.edu/cgi/viewcontent.cgi?article=2428\&context=etds

Ghewy, P. (2010). Guide pratique de l'analyse de données : avec applications sous IBM SPSS Statistics et Excel: questionnez, analysez et... décidez! De Boeck.

Ghssaibi, E. (avril, 2017). Cartable scolaire et devoirs à domicile [Résumé de communication]. Forum : Les curricula libanais : défis et perspectives. CRDP, Beyrouth, Liban. https://www.crdp.org/pdf/uploads/Panel_V_ARABIC_FINAL School equipment and educational tools-MRS.Eva Ghssaibi.pdf

Glasman, D. (2004). Le travail des élèves pour l'école en dehors de l'école (Rapport établi à la demande du Haut conseil de l'évaluation de l'école, $\left.\mathrm{n}^{\circ} 15\right)$. Haut conseil de l'évaluation de l'école. https://www.vie-publique.fr/sites/default/files/rapport/pdf/054000358.pdf

Gomes Magalhaes, M. (2014). La représentation des devoirs à domicile selon parents, enseignants et élèves [Mémoire de bachelor de la Haute école pédagogique BEJUNE, Délémont Suisse]. Rérodoc. http://doc.rero.ch/record/234352

Grawitz, M., (2001). Méthodes des sciences sociales. (11e éd.). Dalloz.

Gruss, A. (2019). L'influence de la famille sur la réussite scolaire à travers l'aide aux devoirs [Mémoire de Master de l'Université Franche-Comté, Besançon, France ]. archives-ouvertes.fr. https://hal-univ-fcomte.archives-ouvertes.fr/hal-02355619/document

Hatab, Z. (2013). Facteurs non éducatifs affectant les deux processus de redoublement et de déperdition scolaires. Le cas de l'école publique au Liban-Cycle de l'éducation de base [Rapport de recherche]. CRDP, Beyrouth, Liban. https://bit.ly/3tl4UdL

Hong, E., Wan, M., \& Peng, Y. (2011). Discrepancies between students' and teachers' perceptions of homework. Journal of Advanced Academics, 22(2), 280-308. DOI: https://doi.org/10.1177\%2F1932202X1102200205

Hoyek, S. (2004). Représentations identitaires et rapport à la formation continue. Cas des enseignants de français au Liban [Thèse de doctorat inédite]. Université Lille 3, France.

Jeynes, W. H. (2007). The relationship between parental involvement and urban secondary school student academic achievement: A meta-analysis. Urban Education, 42(1), 82-110. DOI: https://doi.org/10.1177\%2F0042085906293818

Kalish, N., \& Bennett, S. (2007). The case against homework: How homework is hurting our children and what we can do about it. Crown Publishers.

Kempen, J. (2008). Les travaux à domicile à l'école primaire contribuent-ils à renforcer les inégalités sociales? (Analyse UFAPEC, n 35.08). Union des Fédérations des Associations de Parents de l'Enseignement Catholique. http://www.ufapec.be/files/files/analyses/2008/35-08-devoir.pdf

Kralovec, E. (2014, 5 septembre). Should schools ban homworks? [entrevue]. CNN opinion. CNN. http://edition.cnn. com/2014/09/05/opinion/kralovec-ban-homework/index.html

Kralovec, E., \& Buell, J (2001). End homework now. Educational Leadership, 58(7), 39-43. http://www.ascd.org/ publications/educational-leadership/apr01/vol58/num07/End-Homework-Now.aspx

Lacroix, J. (2012). Alors tes devoirs, c'est fait? (Les analyses de la FAPEO 2012, Analyse 3/15). Fédération des Associations de Parents de l'Enseignement Officiel. https://www.fapeo.be/wp-content/analyses/analyses2012/devoirs.pdf

Meister, G. (2014). La perception des devoirs à domicile chez les enseignants et les parents [Mémoire de bachelor de la Haute école pédagogique BEJUNE, Délémont Suisse]. Rérodoc. http://doc.rero.ch/record/234342

Miedel, W. T., \& Reynolds, A. J. (1999). Parent involvement in early intervention for disadvantaged children: Does it matter? Journal of School Psychology, 37(4), 379-402. DOI: https://doi.org/10.1016/S0022-4405(99)00023-0

Murillo, J., \& Martinez- Garrido, C. (2014). Homework and primary school students' academic achievement in Latin America. International Review of Education, 60(5), 661-681. DOI: https://doi.org/10.1007/s11159-014-9440-2

Centre de Recherche et Documentation Pédagogique- Liban. (2012). Évaluation diagnostique des acquis scolaires (Rapport PASEC Liban, 2012). https://www.crdp.org/files/Rapport\%20Pasec-Liban\%202012.pdf 
Robin, F., Van Lint, S., De Coster, L. et Kahn, S. (2015). Le travail scolaire à domicile. Module de formation sur la question du travail à domicile (rapport commandité par l'OJAJ). Observatoire de l'Enfance, de la Jeunesse et de l'Aide à la Jeunesse. DOI : $\underline{10.13140 / R G .2 .2 .12667 .62240 ~}$

Roderique, T. W., Polloway, E. A., Cumblad, C., Epstein, M. H., \& Bursuck, W. D. (1994). Homework: A survey of policies in the United States. Journal of Learning Disabilities, 27(8), 481-487. DOI: https://doi.org/10.1177\%2F002221949402700803

Rosario, J., Nunez, J., Vallejo, G., Nunes, T., Cunha, J., Fuentes, S. \& Valle, A. (2018). Homework purposes, homework behaviors, and academic achievement. Examining the mediating role of students' perceived homework. Contemporary Educational Psychology, 53, 168-180. DOI: https://doi.org/10.1016/j.cedpsych.2018.04.001

Thlaoui, A. et Biesemans, F. (2020). Les devoirs à domicile: bénéfiques ou toxiques? La Ligue de l'Enseignement et de l'Éducation permanente asbl. https://ligue-enseignement.be/les-devoirs-a-domicile-benefiques-ou-toxiques/

Wilder, S. (2014). Effects of parental involvement on academic achievement : A meta synthesis. Educational Review, 66(3), 377-397. DOI: https://doi.org/10.1080/00131911.2013.780009

\section{Pour citer cet article}

Khatib, W. et Sawli, N. (2021). Les attitudes des enseignants à l'égard des devoirs scolaires au Liban. Formation et profession, 29(1), 1-15. http://dx.doi.org/10.18162/fp.2021.610 